\title{
Empirics for Economic Growth and Convergence
}

\author{
by \\ Danny T. Quah* \\ LSE Economics Department and CEP \\ CENTRE FOR ECONOMIC PERFORMANCE \\ DISCUSSION PAPER NO. 253
}

July 1995

This paper is produced as part of the Centre's Programme on National Economic Performance.

* Comments by J. Dolado, F. Giavazzi, and an anonymous referee have been important in re-shaping this article. X. Sala-i-Martin has generously donated insight and time to try and make me understand. He need not, however, agree with all my statements below. All calculations were performed using the econometrics shell tSrF. 


\section{Nontechnical Summary}

The convergence hypothesis - that poor economies might "catch up"has generated a huge empirical literature: this paper critically reviews some of the earlier key findings, clarifies their implications, and relates them to more recent results. Particular attention is devoted to interpreting convergence empirics. The paper argues that relating them to growth theories, as usually done, gives but one interpretation to convergence dynamics; it does not exhaust their importance. Instead, if we relate convergence to the dynamics of income distributions, it broadens the issues on which such empirics can shed light; it connects with policy concerns on persistent or growing inequality, regional core-periphery stagnation, and tendencies for ongoing capital flows across developed and developing countries. The main findings are: (1) The much-heralded uniform $2 \%$ rate of convergence could arise for reasons unrelated to the dynamics of economic growth. (2) Usual empirical analyses-cross-section (conditional) convergence regressions, time series modelling, panel data analysis - can be misleading for understanding convergence; a model of polarization in economic growth clarifies those difficulties. (3) The data, more revealingly modelled, show persistence and immobility across countries: some evidence supports Baumol's idea of "convergence clubs"; some evidence shows the poor getting poorer, and the rich richer, with the middle class vanishing. (4) Convergence, unambiguous up to sampling error, is observed across US states. 


\author{
Empirics for Economic Growth and Convergence \\ by \\ Danny T. Quah \\ LSE Economics Department and CEP \\ November 1995
}

\begin{abstract}
The convergence hypothesis has generated a huge empirical literature: this paper critically reviews some of the earlier key findings, clarifies their implications, and relates them to more recent results. Particular attention is devoted to interpreting convergence empirics. The main findings are: (1) The much-heralded uniform $2 \%$ rate of convergence could arise for reasons unrelated to the dynamics of economic growth. (2) Usual empirical analyses - cross-section (conditional) convergence regressions, time series modelling, panel data analysis — can be misleading for understanding convergence; a model of polarization in economic growth clarifies those difficulties. (3) The data, more revealingly modelled, show persistence and immobility across countries: some evidence supports Baumol's idea of "convergence clubs"; some evidence shows the poor getting poorer, and the rich richer, with the middle class vanishing. (4) Convergence, una mbiguous up to sampling error, is observed across US states.
\end{abstract}

Keywords: evolving distributions, Galton's fallacy, polarization, regional dynamics, stochastic kernel, unit root

JEL Classification: C21, C22, C23, O41

Communications to: D. T. Quah, LSE, Houghton Street, London WC2A 2AE.

[Tel: +44-171-955-7535, Fax: +44-171-831-1840, Email: dquah@lse.ac.uk] 



\section{Introduction}

It fires the imagination that policy might be able to influence economic growth, thereby allowing poor economies [countries, regions, states, provinces, districts, cities, ... ] either to catch up with those already richer, or to languish, depending. This intellectual excitement is reminiscent of how macroeconomists used to view their ability to stabilize business cycles.

Standard neoclassical models assumed growth to be an inexorable, exogenous process; little then could be said on how growth comes about. More recent theories allow growth to be an endogenous outcome: this, in part, explains renewed interest in long-run macroeconomic behavior, and motivates a research program for empirically discriminating between these two kinds of models in the real world. Over-simplifying drastically, a convenient way to distinguish the two views on growth is to ask, "Are poor economies incipiently catching up with those already richer? Or, instead, are they caught in a poverty trap?" Many caveats would be needed for such an evaluation to be proper, but that catch-up could occur has come to be known as the convergence hypothesis. What is important, though, is that such a hypothesis bears independent interest in economics.

Different kinds of economic convergence are routinely discussed and widely debated. Examples include convergence in incomes between rich and poor parts of the European Union; in plant and firm size in industries; in economic activity across different regions (states, provinces, districts, or cities) within the same country; in asset returns and inflation rates across countries in a common trade area; in political attitudes across different groups; in wages across industries, professions, and geographical regions.

These examples show that convergence is simply a basic empirical issue, one that reflects on-among other things-polarization, income distribution, and inequality. Certainly, understanding economic growth is important. But growth is only one of many different areas in economics where analyzing convergence sheds useful insight. ${ }^{1}$

\footnotetext{
1 Recent applications of convergence-related insights, outside economic growth,
} 
Recent studies on per capita income convergence claim to have uncovered a profound empirical regularity. Poor and rich economies-across different geographical disaggregations; across regions within different countries; across different administrative units; over different time samples-all appear to be converging towards each other at a stable, uniform rate of $2 \%$ per year. This uniformity is striking, and has been used to pronounce on matters as diverse as German reunification; the effects of regional redistribution within individual countries and across the European Union; and the increasing income inequality within countries while the opposite (allegedly) takes place across countries. This paper parallels [35] in reviewing empirical findings on convergence. However, our emphases, interpretations, and criticisms differ sufficiently that the two papers overlap little.

Section 2 begins the analysis by asking if the $2 \%$ convergence-rate uniformity might arise for reasons irrelevant to growth models. The idea here is that such consistency might only reflect something mechanical and independent of the economic structure of growth. The working hypothesis, then, is that economic structure varies in many-explicable and inexplicable-ways across environments, and thus cannot be the source for the $2 \%$ uniformity. Instead, that uniformity is due to something relatively uninteresting, namely, the statistical implications of a unit root in the time series data. Section 2 examines how far such "mechanical" econometric-based explanations can go in explaining conventional empirical findings on convergence. The answer is that they go part of the way, but not all.

Section 3 turns to interpreting convergence dynamics. Here, interest lies partly in the claim that those dynamics shed light on the validity of different growth models. But more direct, and perhaps more important, is the claim that convergence would show the poor catching up with the rich. (This is a growth issue, sure, but not exclusively so.) Does conventional evidence on convergence shed light on

include $[12,13,19,20,28,31]$. Such empirical issues predate $[14,17,27]$, by close to a century, formal endogenous growth models. By coincidence these issues appeared in the industrial organization literature at about the same time as Solow's original growth analysis; see [17] and references. 
this? Section 3 argues that the answer is no: reasons range from Galton's fallacy to researchers' confusing "average" and cross-distribution dynamics. To illustrate further, section 4 presents a growth model whose key observable implications are completely disguised in typical convergence regressions. Whether such a model shows convergence or divergence is a semantic subtlety; this is not normally troubling. In scientific work, mathematical symbols make the intent precise enough. However, most empirical discussions of convergence do not.

Section 5 describes the results from using alternative, more revealing empirics to analyze income data across countries and states. The first key finding is that "convergence clubs" $[4,6]$ are found at the top and bottom of the income distribution across countries: the rich are becoming richer; the poor, poorer; with the middle-class vanishing. The second key finding is that in some-although not all-samples the usual convergence conclusions hold. However, they do so for reasons that are not revealed by those models that are typical in this literature (e.g., all those in [35]). This is for reasons described in Sections 3 and 4: those standard models generate empirics ill-suited for comparison with the dynamics of a rich cross section of data. Section 6 briefly concludes.

\section{Earlier empirical evidence}

Sala-i-Martin [35] compactly summarizes and extends the recent conventional evidence on growth and convergence. ${ }^{2}$ He emphasizes regional dynamics, but the methodological and theoretical discussions apply readily to aggregate economies. Thus this section's difficult work is already done. I simply highlight here some key points in [35] before providing my own critical evaluation of this research.

Integral to these discussions are the concepts of $\beta$ - and $\sigma$-convergence [1]. The striking discovery is the cross-sample stability of rates of $\beta$-convergence. A wide range of examples over different time spans-US states, Japanese prefectures, and different regional European groupings - shows this regularity. The empirical

${ }^{2}$ In this section I assume some basic familiarity with what is known as $\beta$ convergence in this literature; the next section makes things precise. 
fact - that convergence occurs, and does so at the rate of $2 \%$ per annum-comes shining through again and again. Such stability is spectacular and profound, in a profession where very little empirical remains invariant to close scrutiny.

But by the same token one should be skeptical of such findings. How much can one believe growth to be the same across economies, provided only that a few obvious, measurable heterogeneities are removed? And that, further, in that growth process (after conditioning), economies are converging towards the same, unique long-run growth path? Put another way, how credible is it that the simple conditioning used in this literature removes all significant differences in economic growth across economies, so that countries are converging? If not, then why does such apparent uniformity obtain?

Thus, this section critically examines if the $2 \%$ estimate in $\beta$-convergence regressions could arise from a structure completely unrelated to the economics of growth. Finding it so would undermine the claim that that uniformity reflects an interesting economic mechanism. ${ }^{3}$

To understand the discussion that follows, recall the usual derivation of the convergence regression equation. Expositions of this abound, so I need only refer the reader to one of the clearest: equations (1)-(8) in [2]. Equation (8) there is taken to be the important observable implication of the neoclassical growth model. This prediction can be written:

$$
T^{-1}\left(Y_{j}(T)-Y_{j}(0)\right)=a-\left(\frac{1-e^{-\beta T}}{T}\right) Y_{j}(0)+u_{j}(T),
$$

where I use $Y$ to denote $\log$ per capita income, and keep only essential details. Interpret the right hand side of $(2.1)$ as average or long-run growth rate, and

3 The referee has suggested an alternative "mechanical" explanation involving the $\log /$ nonlinear transformations used in $\beta$-convergence regressions. Suggestive computer simulations supporting that conjecture were also generously provided by that referee. I have, however, been unable to provide an analytical explanation for those simulations, whereas I can for mine below. 
$\beta$ as the rate of convergence. The term $u$ is a residual tacked on the end for regression analysis; it is assumed to be uncorrelated with appropriate right-hand side explanatory variables. Thus, equation (2.1) hypothesizes that the average growth rate depends on-among other things - the initial condition $Y_{j}(0)$.

Estimating equation (2.1) by nonlinear least squares, averaging across $j$ in a cross-section-regions, states, countries - constitutes the canonical $\beta$-convergence analysis. An investigator sometimes also groups the time-series observations into blocks of $T$ periods each: panel data methods are then applied to equation (2.1), averaging across both time and cross section. Occasionally, when time series observations have sufficient length, a series of equations (2.1) might be estimated, one for each $j$ : in this case, the investigator averages only across time.

The empirical results in $[1,2,3,35]$ and elsewhere show a remarkable clustering of $\beta$ estimates around a central tendency. That tendency is the magic $2 \%$ rate of convergence. The magic modifier emphasizes this same value's arising from such diverse geographical and time samples. Perhaps it really is the case that the underlying economic structure across countries and regions is invariant. The stability of this $2 \%$-rate would then call for explanation, likely along the lines suggested in [35]. Alternatively, it might be that underlying structures truly differ across time and space, but that enough of a uniformity exists to produce this stability. The question is, Is that uniformity related to convergence dynamics in economic growth?

Recall that a uniformity - well-known in time series econometrics-is borne by unit root stochastic processes. ${ }^{4}$ Fix a time series $X=\{X(t): t \geq 0\}$. While its underlying structure can be quite heterogeneous, as long as it carries a (unit root) stochastic trend, then all other features are eventually subservient to that stochastic trend. I admit this language is a little colorful; it is my interpretation of the following.

4 Unit root time series effects are investigated for economic growth and convergence also in $[7,8]$, although there for different purposes than in this section. 
Theorem: Suppose the time series $X$ is generated by:

$$
\begin{aligned}
& X(t)=b \cdot X(t-1)+\epsilon(t), \quad t \geq 1 ; \\
& \quad b=1 ; \text { and } \\
& X(0) \text { an arbitrary random variable. }
\end{aligned}
$$

Provided that $\epsilon$ satisfies weak regularity conditions, the OLS coefficient in a regression of $X$ on itself lagged once converges in probability to 1 .

This result can be proved in a number of different ways and with a number of different meanings assigned to "weak regularity conditions". A clear and insightful exposition is in [25].

As in $\beta$-convergence empirical results, this Theorem provides an invariance. The disturbances $\epsilon$ can be (relatively) arbitrary without affecting the conclusion. They can have different variances. They can be serially correlated; any stationary ARMA process for $\epsilon$ is allowed. None of these changes the conclusion.

Embedded in the casual statement that $\epsilon$ 's can be serially correlated is of course when $\epsilon(t)$, the regression disturbance, is correlated with $X(t-1)$, the regressor. In usual regressions this would bias the OLS estimator for $b$ at all sample sizes including infinity. However, when $b$ has the special value of 1 , none of these matters. None of these "problems" alters the result that $b$ 's OLS estimator converges to its correct value of unity.

How is this relevant for the current discussion? Suppose that the differences across countries, regions, states, and time appeared only in variables omitted from the regression; these variables would be analogous to heterogeneous $\epsilon$ 's. Then, those differences might be large and interesting, but all that the investigator could hope to uncover is the unit coefficient of the Theorem. Such an invariant unit coefficient might well be interesting for time-series econometrics, but is it what convergence researchers wish to uncover? How does such invariance relate to the economics of the convergence hypothesis? Not at all, I suspect, although an unwary researcher might conclude that the empirical stability shows something profound. 
Is it merely idle speculation that connects the Theorem's invariance with the stability of $\beta$ in convergence regressions? After all, those regressions aren't exactly the regressions described by the Theorem. But, then again, how different are they?

Equation (2.1) can be rearranged as:

$$
\begin{aligned}
T^{-1} Y_{j}(T) & =a+e^{-\beta T} \cdot T^{-1} Y_{j}(0)+u_{j}(T) \\
& =a+b_{T} \cdot T^{-1} Y_{j}(0)+u_{j}(T),
\end{aligned}
$$

where I have defined $b_{T}=e^{-\beta T}$. Absorb $T^{-1}$ into both regressor and regressand. Equation (2.2) looks not all that different from the model in the Theorem. When $\beta$ is 0.02 , and $T$ is 10 , the coefficient $b_{T}$ equals 0.82 ; when $T$ is $20, b_{T}$ is $0.67 ; T$ $30, b_{T} 0.55 ; T 40, b_{T} 0.45 ; T 50, b_{T} 0.37 ; T 100, b_{T}$ 0.14. Evidently, when $\beta$ is 0.02 , the coefficient $b_{T}$ does vary and is nowhere near unity for time intervals of interest. But notice, however, that when $\beta$ is 0.02 and $T$ is 1 , then $b_{T}$ is 0.98 . This is reasonably close to the unit root in the Theorem. ${ }^{5}$ Perhaps $\beta$-convergence regressions are simply unit-root regressions in disguise; perhaps the stability of estimated convergence rates simply reflects the invariance in the Theorem. Is this cause for worry?

Table 1 below presents results from a small Monte Carlostudy, designed to see if a unit root in $Y$ can reproduce the literature's key findings, not just analytically as above, but in samples comparable to those used in practice. The calculations in the table mimic those used by convergence researchers.

Some preliminary comments are in order. Following the practice in this literature, $\beta$ is estimated by nonlinear least squares. Since equation (2.2), or equivalently (2.1), leaves $a$ and $\beta$ exactly identified, why not estimate $a$ and $b_{T}$ by ordinary least squares, and then find $\beta$ as $-T^{-1} \log \left(b_{T}\right)$ ? As anyone who has done Monte Carlo experimentation can attest, nothing guarantees that, whatever the true datagenerating model, the OLS estimate for $b_{T}$ is positive. When a negative value for

5 Unit roots regression theory also says that with finite $T$, the OLS estimator is typically biased downwards-this meshes nicely with the 0.98 value required for $b_{1}$. 
$b_{T}$ occurs, $\beta$ is undefined. Estimating $\beta$ by nonlinear least squares in the form of either equations (2.2) or (2.1) rescues the investigator from this embarrassment.

Doing so, however, introduces a different problem: when the best-fitting $b_{T}$ is negative or zero, any good nonlinear least squares program will try to give $\beta$ a value of infinity. Thus in finite samples very large positive values for $\beta$ can be generated in Monte Carlo draws, thereby pulling rightward the estimator's simulated distribution. This then gives Monte Carlo averages for convergence rates that are even faster than $2 \%$ - even when the true data-generating model comprises cross-sectionally independent random walks, and thus shows no convergence. ${ }^{6}$

To remove this bias in my favor (since the immediate assignment is to cast doubt on convergence findings), I have trimmed the Monte Carlo distributions for $b$-estimators at machine accuracy for the exponential function. ${ }^{7}$ Table 1 presents results for a variety of time-series lengths $T$ and cross-section sizes $N$. Each column block, under a given value of $T$, contains three numbers for each value of $N$. The first number is the average $\beta$ in the trimmed Monte Carlo distribution; how close this is to $2 \%$ shows how well the conjectures above hold up. The second number (in parentheses) is the cross-Monte Carlo sample standard deviation (not the estimated standard error from nonlinear estimation): this is the appropriate notion of imprecision in our experiment. Finally, the third number [in brackets] denotes the fraction of draws rejected in trimming the Monte Carlo distribution; this number is smaller for larger $N$ and $T$, reflecting our estimator's convergence in probability to the correct, well-defined parameter value. ${ }^{8}$ The true data-generating process in

${ }^{6}$ Of course, this problem goes away as the sample gets arbitrarily large, but for that we already have the analytical results above. It is precisely the small-sample case that we currently need to consider.

7 Thus, my tSrF program generating the table will produce slightly different results across different machines. The NeXT that I use probably has slightly better accuracy than most other personal computers.

${ }^{8}$ Large $N$, large $T$ analysis can be found in, e.g., [29]. The convergence in probability here follows easily from that analysis. 
the experiment comprises cross-sectionally independent Gaussian random walks, each with innovation variance $T^{-1}$. (Even in the small samples here, the estimates are already roughly invariant to the choice of innovation variance.)

Look down the table's columns: for moderate-sized samples, $2 \%$ convergence rates are not at all atypical - even when the true model comprises mutually independent random walks, and thus the true convergence rate is 0 . Our reasoning says this is nothing more than finite-sample bias. It therefore reconciles two seemingly contradictory facts described in [24, pp. 255-6]: one, a theoretical prediction on divergence of independent random walks; and the other, Barro and Sala-i-Martin's $\beta$-convergence findings. The table's underlying sample is exactly a collection of cross-sectionally independent random walks. Despite this, $\beta$-convergence is rampant. For example, in samples where the cross-section and time-series dimensions are both 50 the average estimated convergence rate is $4 \%$. When we retain the time-series dimension, but increase the cross-section to 100, the average estimated convergence rate becomes $2 \%$. Keeping the cross-section dimension at 50 , but increasing the time-series dimension to 60 , the average becomes $1.4 \%$. As both $N$ and $T$ increase, the table shows the bias (away from zero) vanishing. The theoretical analysis in [29] suggests this occurs at rate $T^{-1}$ when $T$ increases but only at rate $N^{-1 / 2}$ as $N$ grows. Table 1 shows precisely such behavior.

What do we conclude from this? If we were to focus on just the means of the estimates, we would start to doubt the credibility and interpretation of $\beta$ convergence findings. Noting that Table 1's results are approximately invariant to additional heterogeneity only strengthens those doubts. However, the Monte Carlo distributions also endow the estimates with high variability (the numbers in parentheses), much higher than $\beta$-convergence researchers usually find. Our mechanistic explanation, therefore, cannot be the entire story. If all the empirical findings on $\beta$-convergence had came out of one very large meta-draw on histories around the world, and that meta-draw had realized at its mean value, then the calculations and mechanisms above could well explain published $\beta$-convergence results. In my view, that is unlikely. Nevertheless, the small sampling experiment 
does suggest that part of the explanation could be merely a statistical invarianceinteresting from the view point of econometric theory, less so from the perspective of economic convergence.

\section{Convergence predictions in growth theory}

Many studies of convergence empirics still share the same theoretical motivation as that given in [2]. Recall how dynamics are analyzed there: one log-linearizes the deterministic Cass-Koopmans model about steady state, and then notices that the growth rate of output per worker depends on the $\log$ deviation from steady state.

This dependence is parameterized, as usual, by a negative eigenvalue of a firstderivatives matrix. In the model that eigenvalue varies with the ratio of physical capital's marginal to average product, or equivalently, when factor markets are competitive, physical capital's factor share in income. The larger is that ratio or factor share, the closer is the relevant eigenvalue to zero, and thus, the slower is the rate of convergence.

Barro and Sala-i-Martin's $[1,2]$ estimated $\beta$-convergence rate implies capital factor shares larger than the 0.4 given in national income accounts. The same convergence estimate also says physical capital enters the production function importantly (has marginal product that doesn't diminish quickly; see, e.g., [34]). In this analysis the two - estimated convergence rates and physical capital's compensation - together create a puzzle for neoclassical growth theory. For some economists, it is this and only this that justifies interest in convergence empirics.

Others will quickly disagree-even before questioning the accuracy of the estimated numbers. Most obvious, if the researcher's interest lies only in the coefficients of a production function, then why not just estimate those directly? A long and revered tradition in empirical analysis - associated with [16] and many others-treats exactly that estimation problem. Starting from there, and refining estimates, would appear sensible if one were interested in parameters in a production function. Using the dynamics of state, regional, or cross-country data only 
to make a point about a coefficient in a Cobb-Douglas production function (say) seems circuitous, although creative.

More tenable might be to recall the independent interest in convergence: whether poorer economies are incipiently catching up with richer ones is a basic question, and of considerable interest. Relating the question to the parameters of a neoclassical production function merely places an interpretation on it; it does not exhaust its importance. Answering this broader convergence question would directly address issues raised in [4]. It would speak to the concerns of European Commission policy-makers analyzing inequality (divergence) across European regions [5]. Finally, addressing the question this way brings into the (macroeconomic) analysis ideas and insights from the economics of income distributions. ${ }^{9}$ The coupling of the convergence hypothesis to these broader issues is, arguably, what animates debate here-more than does concern with just the parameters of an aggregate production function.

The discussion that follows will be easier to understand if one keeps in mind these broader issues. Doing so immediately clarifies the usefulness of different concepts of convergence. ${ }^{10}$ To begin, recall Barro and Sala-i-Martin's $[1,2] \beta$ - and $\sigma$-convergence.

Roughly put, $\beta$-convergence is when in a cross-section regression of (timeaveraged) growth rates on initial levels, the coefficient on initial levels is negative: "poorer regions grow faster." Conditional $\beta$-convergence is again a negative coefficient, but only when that regression has the appropriate, additional explanatory

9 Distinguish this, however, from work such as [15]. That work focuses on the relation between aggregate growth and the distribution of income across people within an economy. Here, instead, I refer to using income distribution ideas to model dynamics across many economies.

10 One shouldn't really need to say this here (except that misunderstandings should be corrected earlier rather than later): the issues here differ from the usual probabilist's distinction between convergence in probability, in law, almost sure, in $L^{p}$, and so on. 
variables on the right hand side. While potentially important in practice, for the discussion here the difference between conditional and unconditional convergence adds no conceptual insight. Thus, I will not mention conditional convergence further in this section; in the next I raise it, but only to indicate how it confuses issues.

Again, roughly put, $\sigma$-convergence is when the dispersion of cross-section levels diminishes over time. Typically, dispersion is measured by sample standard deviation. Here, it is irrelevant whether a single country shows convergence (in mean square) to steady state or to anything else: what matters, instead, is how the entire cross-section behaves.

The literature (e.g., $[1,35]$ ) has already explored some of the relation between $\beta$ - and $\sigma$-convergence. Here, I give precise definitions, and study the connection more fully; this will also help motivate the dynamically evolving distribution empirics to be given below.

Let $Y_{j}(t)$ denote-as above- the $\log$ of economy $j$ 's income at time $t$. Say that the data

$$
Y=\left\{Y_{j}(t): j=1,2, \ldots, N ; t=0,1, \ldots, T\right\}
$$

show $\beta$-convergence if for all $t$ the cross-section projection

$$
P\left(Y_{j}(t)-Y_{j}(0) \mid Y_{j}(0)\right) \stackrel{\text { def }}{=}\left(b_{t}-1\right) \times Y_{j}(0),
$$

has

$$
b_{t}-1<0
$$

Let $\sigma_{t}$ denote the $(N$-sample-size $)$ cross-section standard deviation, i.e.,

$$
\sigma_{t}=\left(N^{-1} \sum_{j=1}^{N}\left[Y_{j}(t)-\left(N^{-1} \sum_{k=1}^{N} Y_{k}(t)\right)\right]^{2}\right)^{1 / 2} ;
$$

and say that data $Y$ show $\sigma$-convergence if $\sigma_{t} \leq \sigma_{t-1}$ for all $t$. Some might insist on $\sigma_{t} \rightarrow 0$ as $t$ grows large but that will never be observable, and so is 
not a useful criterion in practice. Also, the inequality is weak rather than strict to allow situations where $\sigma_{t}$ has already converged, i.e., the cross-section is already in (stochastic) steady state. Even when $\sigma_{t}$ is unchanging through time, the economies underlying the cross section could still be moving about within that invariant distribution: this is, after all, what "stochastic steady state" means.

Current interest lies only in the relation between different concepts of convergence. Thus, sampling variability is inessential in the discussion; I will ignore it in manipulating (3.1) and (3.2).

Begin with an extreme special case. Suppose $Y$ 's are independent and identically distributed (iid) cross-sectionally, and in time follow:

$$
\begin{gathered}
Y_{j}(t)=b Y_{j}(t-1)+u_{j}(t), \quad|b|<1, \\
Y_{j}(0) \text { independent of } u_{j}(t), \quad t \geq 1,
\end{gathered}
$$

where $u$ is iid also in time, and has positive, finite variance $\sigma_{u}^{2}$. (That $Y$ has been assumed iid across $j$ implies $u$ iid across $j$.)

Equation (3.3) implies that

$$
\sigma_{t}^{2}=b^{2} \sigma_{t-1}^{2}+\sigma_{u}^{2} \Longrightarrow \lim _{t \rightarrow \infty} \sigma_{t}^{2}=\left(1-b^{2}\right)^{-1} \sigma_{u}^{2}
$$

and

$$
b_{t}=b^{t} \Longrightarrow b_{t}-1<0 \text { for all } t .
$$

Thus, (3.3) shows $\beta$-convergence. Whether it also shows $\sigma$-convergence depends on whether $\sigma_{0}^{2}$ is greater than $\left(1-b^{2}\right)^{-1} \sigma_{u}^{2}$. This is a restriction on the initial condition $Y_{j}(0)$. If $\sigma_{0}^{2}>\left(1-b^{2}\right)^{-1} \sigma_{u}^{2}$, then $\sigma_{t}^{2}$ falls over time monotonically towards its limiting value. If, however, $\sigma_{0}^{2}<\left(1-b^{2}\right)^{-1} \sigma_{u}^{2}$ then $\sigma_{t}^{2}$ has to grow, and $Y$ would then not show $\sigma$-convergence. This is what Barro and Sala-i-Martin [2, pp. 227-8] mean by $\beta$-convergence not implying $\sigma$-convergence. Figure 3.1 shows this: all economies began close together, and over time approach a cross-section distribution with $\sigma_{t}=\left(1-b^{2}\right)^{-1} \sigma_{u}^{2}$. (For now, ignore the arrows criss-crossing in the right-most part of the picture.) 
The situation when $\beta$ - and $\sigma$-convergence coincide is presumably that which most researchers would like. One can then identify $\beta$-convergence in a regression like equation (3.1) with poor economies' catching up alongside the rich. Figure 3.2 illustrates exactly that. In such a picture it is most natural to suppose the initial cross-section distribution to be due to a large, one-time perturbing disturbance, say, World War 2 or German reunification or the sudden accession of many countries to the European Union. The subsequent transition dynamics then reflect the workings of the neoclassical growth model-I have also indicated this, suggestively, in the picture.

The discussion leading to figure 3.1 has already cautioned that $\beta$-convergence does not-in theory-imply dynamics like those in figure 3.2. Thus, the finding of a $2 \%$ rate of convergence does not imply, say, East Germany's catching up to West Germany at that rate. The separation between them, as measured by the spread $\sigma$, need not be decreasing, even when $\beta$-convergence obtains.

The uninformativeness of $\beta$-convergence is, up until now, only a theoretical possibility. Can one say if in practice such considerations matter? Figure 3.2 suggests they would be irrelevant if reality is the tracing out of transitory dynamics following one large, displacing disturbance-what paleontologists call a punctuated equilibrium. In [26], I give evidence against this view: for the post-War period, disturbances to the cross-country distribution of incomes are ongoing. They are not one-shot, occurring only at the beginning of the sample; instead, over this period, the evidence suggests that disturbances are likely increasing in severity through time. Thus, for analyzing cross-country growth dynamics the punctuated equilibrium concept appears problematic. And what about across regions, states, or economies smaller than countries? There is little reason to expect anything different.

The conclusion above can be re-stated a little more generally: $\beta$-convergenceor more generally looking at coefficients in a cross-section regression-is uninformative for a distribution's dynamics. Cross-section regressions can represent only 
average behavior, not the behavior of an entire distribution. ${ }^{11}$

Going over the reasoning above, a reader might conclude that looking at $\sigma$ convergence would get around all these difficulties. Whether $\beta$-convergence implies $\sigma$-convergence or not then becomes irrelevant: the focus will have moved to $\sigma$ convergence directly. This, obviously, solves the previously-mentioned problems. But it introduces new subtleties.

From observations already noted above, disturbances to the cross section of economies should be viewed as ongoing over time. If so, then one is not going to see $\sigma_{t}$ tending to zero, but at best to a positive constant. Go to that limit: the right hand side of figure 3.1 shows this, but figures 3.3 and 3.4 make clearer a couple of possibilities. In figure 3.3 one should imagine there being many, many economies - the figure explicitly draws time paths for only two of them. The world according to figure 3.3 has economies criss-crossing and leap-frogging: there is substantial intra-distribution dynamics. While all that is happening, $\sigma_{t}$ remains unchanged. It is such dynamics that appeared in the right-most portion of figure 3.1. Figure 3.4 also shows a world with $\sigma_{t}$ invariant. But here, unlike in figure 3.3, there is persistent inequality: rich economies always remain rich; poor ones, poor. Studying just $\sigma_{t}$ 's - one characteristic of the cross section distribution-does not distinguish between the two worlds in figures 3.3 and 3.4.

These examples are, of course, extreme and unrealistic, but they make transparent the different effects that might be at work in reality. Whether the world is better described by the dynamics in figure 3.3 or figure 3.4 certainly matters for our basic question, are the poor catching up with the rich? We conclude that, as with $\beta$-convergence, the empirics of $\sigma$-convergence cannot deliver, even in theory, a useful convincing answer. Figures 3.1-3.4 clarify that the problem of analyzing convergence is exactly that of analyzing the dynamics of evolving distributions. For convergence one is interested in how one part of the distribution behaves relative to another: that is, after all, what "catch-up" means. Any empirical technique

11 An early example of this important conceptual distinction is Galton's fallacy of regression towards the mean $[14,17,27]$. 
not recognizing this can only be stopgap at best.

Time series methods are clearly uninformative here if those methods do not acknowledge that it is features of the cross section distribution that are of interest. Instead, typically, time-series researchers might use the extensive cross section information here only by averaging across countries, thereby obtaining more precise estimates. Such methods can say nothing about the behavior of one part of the distribution relative to another. Alternatively, the different economies in the cross section might be stacked into a vector time series; this could then be informative on how certain parts of the cross section evolve relative to others. However, the researcher is then left with the problem of modelling a vector whose dimension exceeds the number of time series observations on it.

The preceding discussion is sometimes interpreted as arguing for combining cross-section and dynamic information to study convergence. In that view, crosssection regressions are thought to exploit the first kind of variation, but to neglect the second; time series methods, to do the opposite. This idea leads to using panel data methods, thereby combining cross section and dynamic variation. But, of course, this raises concern over the "problem" of individual (fixed or random) effects. The plethora of techniques for dealing with that problem is then dragged out and applied. But does this solve any of the problems described above?

The answer is no. As with ordinary cross-section regression, panel data techniques will, again, only capture "representative" economy dynamics, not those of the entire distribution. To see this, simply ask, Can those techniques say anything about how the poorest $10 \%$ of the distribution are catching up with the richest $10 \%$ ? No, they are silent on this. Worse, using the panel structure to take care of the individual effects only exacerbates the difficulties. Whether one models those individual effects as fixed or random or anything else, one simply sweeps out and keeps unexplained the persistent differences across economies.

It is crucial to remember the economic problems that panel data techniques are meant to solve. Chamberlain [9] provides a clear discussion of those; they differ from the problems here. Simply because panel data techniques happen to apply 
to data with extensive cross-section and time-series variation does not mean they are at once similarly appropriate for analyzing convergence.

\section{An economic model: Conditional and unconditional convergence}

The preceding analysis drew some pretty pictures. It then suggested that the dynamics in those pictures cast doubt on the informativeness of standard growth regressions. But is there an economic structure that could produce such dynamics? This section describes a model that does so.

It is standard in many growth analyses to study how one economy, in isolation from all other economies, grows quickly or slowly. ${ }^{12}$ The insights developed are then used to explain why some countries grow faster than others.

The point made in the previous section bears on this and, with hindsight, is obvious: studying an average or representative economy gives little insight into the empirical behavior of the entire cross section. For such cross section dynamics to be interpretable, one needs a theoretical model that makes predictions on them.

The model here draws from [30]. It makes predictions on cross section dynamics by linking three observations: (i) countries endogenously select themselves into groups, and thus do not act in isolation; (ii) specialization in production allows exploiting economies of scale; and (iii) ideas are an important engine of growth.

The key results are two: (a) coalitions - or, as it will turn out, convergence clubs-form endogenously; the model delivers predictions on coalition membership across the entire cross section of economies; (b) different convergence dynamics are generated, depending on the initial distribution of characteristics across countries. Included among these potential dynamics are explicit convergence club characterizations; polarization - the rich becoming richer, the poor poorer, and the middle class vanishing; stratification-multiple modes in the income distribution across countries; and overtaking and divergence-two economies initially on roughly equal footing, separating over time so that one eventually becomes wealthier than the other.

12 Exceptions do exist, e.g., [18, 21]. 
In [30] I show that coalitions, in equilibrium, are typically nondegenerate, nontrivial subsets of the entire cross section of economies. The configuration of coalitions comes from recognizing the forces across countries for consolidation and fragmentation. Equilibrium balances these opposing forces, and permits (at least for a time) diversity. Coalitions can then be recognized as "convergence clubs," thereby determining international patterns of growth, convergence, and polarization.

The resulting dynamics are in general subtle, but special cases can be graphically represented. Figure 4.1 shows some equilibrium time paths for incomes (discounted by the steady-state growth rate).

In this example, at time $t_{0}$ there is some initial income distribution across the cross section of economies. Over time, some economies become better off, others worse off; overtaking is possible. Coalitions or convergence clubs form, however, and the distribution tends towards a bimodal distribution at time $t_{1}$. In general, the number of modal points equals the number of coalitions that form. When more than two coalitions form, stratification is an apposite term in place of polarization to describe the outcome. With two coalitions (as in figure 4.1), the distribution dynamics can be easily seen. Eventually, the middle-income group of economies vanish, and the rich continue to become richer, and the poor, poorer. Clustering occurs at high and low parts of the income distribution.

The exact outcome- the number of coalitions, their composition, and so ondepends on the initial distribution of income across the entire cross section. If the world began with all incomes already close together, then only a single coalition forms; all countries then converge to equality. If, on the other hand, initial incomes are disparate, then, more likely, multiple convergence clubs form. The distribution dynamics will then be the multi-mode or stratification extension of figure 4.1. Economists observing these dynamics are naturally led to describe the resulting groups as convergence clubs.

But what happens if researchers apply the tools of $\beta$-convergence analysis to data generated by figure 4.1? A researcher might attempt to understand the 
behavior of incomes across the cross section of economies by, say, "controlling" for differing human capital stocks and other observable variables. That researcher could then conclude that conditional convergence occurs, and that human capital explains cross-country patterns of growth. However, such conclusions mislead. It is, instead, the pattern of club membership that explains everything. In the model, human capital is only responding endogenously to coalition structures: that is why high human capital is found among rich-club countries. Moreover, conditional convergence is not a useful way to think about the polarization induced by convergence-club formations: the interesting stratification in figure 4.1 is never revealed by conditional-convergence investigations.

The dynamics in figure 4.1 would be one motivation for the empirics developed in [11]. There, Durlauf and Johnson provide an innovative technique for consistently uncovering local basins of convergence - they do this by allowing their fitted regression model to "adapt" subsamples, depending on the data realizations themselves. What I give below is a different empirical method, but one that seems to me more natural for studying evolving distributions. Durlauf and Johnson interpret their empirics in terms of multiple regimes. I interpret figure 4.1 as just one equilibrium law of motion, but in an entire distribution, which could then have multiple modal points in the ergodic limit. In general, each technique - that in [11] and the section below-has advantages in different dimensions over the other; neither strictly dominates.

Finally, the empirical message here is not confined to this particular model of ideas and growth. In [33] I adapted the model in [15] to produce much the same empirical results as in figure 4.1. Other models employing local nonconvexities in the technology, nonlinearity in the savings function, and so on will, again, give the same empirics (see, e.g., [6]). 


\section{Revealing empirics}

The analysis above has argued that standard convergence empirics are uninformative. This section describes some newer empirical results that overcome those deficiencies.

From figure 4.1 it is obvious that the natural way to study convergence empirics is to provide an empirical model for how distributions evolve. ${ }^{13}$ Let $F_{t}$ denote the distribution of incomes across countries at time $t$. Associated with such a distribution is a measure $\lambda_{t}$. The simplest model for the evolution of $\left\{F_{t}\right.$ : integer $\left.t\right\}$, or equivalently $\left\{\lambda_{t}\right.$ : integer $\left.t\right\}$, is an autoregression in measures:

$$
\forall \text { measurable sets } A: \quad \lambda_{t+1}(A)=\int M(y, A) d \lambda_{t}(y),
$$

where $M$ is a stochastic kernel, mapping the Cartesian product of income values and measurable sets to the interval $[0,1]$. The kernel $M$ maps one measure $\lambda_{t}$ into another $\lambda_{t+1}$, and tracks where in $F_{t+1}$ points in $F_{t}$ end up. Thus, $M$ encodes information on intra-distribution dynamics, whether economies like Korea and the Philippines, say, which were close together in 1950 transit subsequently to widely different income levels. It therefore contains strictly more information than just aggregate statistics such as means or standard deviations.

Equation (5.1) is analogous to a standard time-series first-order vector autoregression, except its values are distributions (rather than scalars or vectors of numbers), and it contains no explicit disturbance or innovation. By analogy with autoregression, there is no reason why the law of motion in $\lambda_{t}$ need be first order, or why the relation need be time-invariant. Nevertheless, $(5.1)$ is a useful first step for analyzing dynamics in $\left\{\lambda_{t}\right\}$. Rewrite (5.1) as the convolution

$$
\lambda_{t+1}=M * \lambda_{t} .
$$

13 The ideas that follow were first stated in a simpler form in [26]. More precise technical statements can be found in [30, 32]. 
Iterating (5.2) yields (a predictor for) future cross section distributions

$$
\lambda_{t+s}=(M * M * \cdots * M) * \lambda_{t}=M^{s} * \lambda_{t}
$$

taking this to the limit as $s \rightarrow \infty$, one can characterize the likely long-run or ergodic distribution of cross-country incomes. Convergence towards equality might manifest in $\left\{\lambda_{t+s}\right\}$ tending towards a degenerate point measure; the world polarizing in the long run as in figure 4.1 might manifest in $\left\{\lambda_{t+s}\right\}$ tending towards a two-point or bimodal measure. The speed of convergence of the evolving distributions and their cross-sectional mobility properties can be studied from certain spectral characteristics of the kernel $M$. Variants of (5.1) thus allow answering a wealth of interesting questions about cross-sectional income dynamics.

How does one estimate something like $M$ ? The most natural first step is to discretize the measures $\lambda_{t}$. Thereupon, $M$ becomes just a transition probability matrix; and $\lambda$ 's become nonnegative vectors on the unit simplex. Of course, discretization distorts the underlying model; in Markov process theory, it is well known that a first-order Markov process need no longer be even Markov when inappropriately discretized. However, one suspects that for effects such as those in figure 4.1 the distortions will not conceal the important features.

Quah [26, 27] has calculated discretized estimates of $M$ for the world's crosscountry distribution of per capita incomes. Table 2 reproduces one such result from [26]. Taking each country's per capita GDP relative to the world average, discretize the set of possible values into intervals at $1 / 4,1 / 2,1$, and 2 . All relevant properties of $M$ are then described by a $5 \times 5$ transition matrix whose $(j, k)$ entry is the probability that an economy in income group $j$ transits to income group $k$. Low-numbered states correspond to low incomes; thus, for example, income group 1 (the first row and column) in table 2 comprises per capita incomes no greater than one-fourth the world's average.

In table 2 the column labeled (Number) gives the total number of transitions that have starting points in that income group. For example, the second row shows that over the entire sample-across 118 countries and 23 years - 643 observations 
fell in income group 2, i.e., started with incomes between one-fourth and onehalf the world average. Of these, $92 \%$ remained in that same income group the following period.

Table 2 presents kernel $M$ where the transition period is one year. The predominant feature is - not surprisingly - high persistence: all diagonal entries exceed $90 \%$; other entries are non-zero only for the first state off the main diagonal. More interesting, however, is the final row, which tabulates the ergodic distribution implied by the estimated kernel. ${ }^{14}$ That distribution shows first, a thinning in the middle, and second, an accumulation in both low and high tails. But these are exactly the important features suggested in figure 4.1: it is polarization that occurs across the world, not convergence. Convergence clubs exist at the high and low ends of the income distribution; the middle class is vanishing. (These tendencies are also suggested informally by figure 6 of $[27$, p. 436].)

Table 3 presents the same calculations, but now for US states. Here, much greater mobility is apparent: diagonal entries are smaller; off-diagonals, larger. The evidence therefore suggests greater convergence occurring within the states of the US. Also, note the ergodic distribution: it no longer shows the bimodality in rich and poor that is evident in world income distribution dynamics. No convergence clubs or clusters are evident here.

In other papers $[26,27,28,32,33]$ I have studied variations on the basic theme here, namely, that empirical analysis of convergence is more revealing when it provides information on how the entire distribution evolves - not just the dynamics of a representative economy. ${ }^{15}$ Included among these variations are: allowing higher-order dynamics than first-order as in equation (5.1); taking natural time horizons to be greater than one year; allowing the grid-points in tables 2 and 3 to adapt and evolve over time; not discretizing, but instead retaining the continuous

14 Nothing in the calculations enforces existence or uniqueness of an ergodic distribution. It is a consequence of the data that precisely one such distribution was found.

15 Other subsequent, recent work following up on these ideas include [10, 22, 23]. 
set of income values (in which case the kernel $M$ becomes infinite-dimensional, and is estimated nonparametrically); permitting conditioning information; structuring $M$ so that it can be more easily interpreted. None of these alters the principal finding on polarization for the world cross-country distribution of incomes.

\section{Conclusion}

This paper has provided theoretical and empirical frameworks for studying convergence. In doing so, it has overcome some difficulties in the traditional analysis of, e.g., $[2,35]$.

The first point the paper made is that it is possible, although not likely, that standard convergence findings are due to an uninteresting (from the current perspective) statistical uniformity. But then, second, the paper provided a rich array of examples to argue that those convergence findings might be misleading.

Next, the paper described a theoretical model of ideas and economic growth. In the model, convergence clubs endogenously form, and the distribution of income across economies polarizes. Rich economies become richer; poor ones, poorer; and the middle class vanishes. Such a model produces equilibrium dynamics where conventional empirical methods are problematic. The model suggests, instead, alternative empirics based on studying the dynamics of evolving distributions.

The paper then gave results from such empirical analyses, suggesting the strength of cross-country polarization present in the world. The paper argued that that kind of divergence actually dominates the evidence on $2 \%$ convergence, previously accepted as conventional wisdom. 


\section{References}

[1] Barro, Robert J. and Sala-i-Martin, Xavier. (1991), "Convergence Across States and Regions", Brookings Papers on Economic Activity, 1:107-182, April.

[2] Barro, Robert J. and Sala-i-Martin, Xavier. (1992), "Convergence", Journal of Political Economy, 100(2):223-251, April.

[3] Barro, Robert J. and Sala-i-Martin, Xavier. (1992), "Regional Growth and Migration: A Japan-United States Comparison", Journal of the Japanese and International Economies, 6(4):312-346, December.

[4] Baumol, William J., Blackman, Sue Anne Batey, and Wolff, Edward N. (1989), Productivity and American Leadership: The Long View, MIT Press, Cambridge MA 02139.

[5] Begg, Iain and Mayes, David. (1993), "Cohesion in the European Community", Regional Science and Urban Economics, 23:427-448.

[6] Ben-David, Dan. (1994), "Convergence Clubs and Diverging Economies", Working Paper 922, CEPR, London W1X 1LB, February.

[7] Bernard, Andrew B. and Durlauf, Steven N. (1993), "Interpreting Tests of the Convergence Hypothesis", Working paper, MIT Economics Department, December.

[8] Bernard, Andrew B. and Durlauf, Steven N. (1994), "Convergence in International Output", Working paper, MIT Economics Department, February.

[9] Chamberlain, Gary. (1984), "Panel Data", in Griliches, Zvi and Intriligator, Michael D., (eds.), Handbook of Econometrics vol. II, chapter 22, pages 12471318. Elsevier North-Holland, Amsterdam.

[10] Desdoigts, Alain. Changes in the World Income Distribution: A NonParametric Approach to Challenge the Neoclassical Convergence Argument. PhD thesis, European University Institute, Florence, June 1994. 
[11] Durlauf, Steven N. and Johnson, Paul. (1994), "Multiple Regimes and CrossCountry Growth Behavior", Working paper, University of Wisconsin, May.

[12] Eckstein, Zvi and Eaton, Jonathan. (1994), "Cities and Growth: Theory and Evidence from France and Japan", Working paper, Economics Department, Tel-aviv University, September.

[13] Esteban, Joan-María and Ray, Debraj. (1994), "On the Measurement of Polarization", Econometrica, 62(4):819-851, July.

[14] Friedman, Milton. (1992), "Do Old Fallacies Ever Die?", Journal of Economic Literature, 30(4):2129-2132, December.

[15] Galor, Oded and Zeira, Joseph. (1993), "Income Distribution and Macroeconomics", Review of Economic Studies, 60(1):35-52, January.

[16] Griliches, Zvi and Ringstad, Vidar. (1971), Economies of Scale and the Form of the Production Function, North-Holland, Amsterdam.

[17] Hart, Peter E. (1994), "Galtonian Regression Across Countries and the Convergence of Productivity", Discussion Paper 19, University of Reading, March.

[18] Helpman, Elhanan. (1993), "Innovation, Imitation, and Intellectual Property Rights", Econometrica, 61(6):1247-1280, November.

[19] Konings, Joep. Gross Job Flows and Wage Determination in the UK: Evidence from Firm-Level Data. PhD thesis, LSE, London, July 1994.

[20] Koopmans, Reinout and Lamo, Ana R. (1994), "Cross-Sectional Firm Dynamics: Theory and Empirical Results from the Chemical Sector", Working paper, Economics Department, LSE, London, April.

[21] Krugman, Paul. (1979), "A Model of Innovation, Technology Transfer, and the World Distribution of Income", Journal of Political Economy, 87(2):253-266, April.

[22] Lamo, Ana R. (tba). PhD thesis, LSE, London, 1995. in progress. 
[23] Larch, Martin. (1994), "Regional Cross-Section Growth Dynamics in the European Community", Working paper, European Institute, LSE, London, June.

[24] Lucas Jr., Robert E. (1993), “Making a Miracle”, Econometrica, 61(2):251271 , March.

[25] Phillips, Peter C. B. (1987), "Time Series Regression with Unit Roots", Econometrica, 55(2):277-302, March.

[26] Quah, Danny. (1993), "Empirical Cross-Section Dynamics in Economic Growth", European Economic Review, 37(2/3):426-434, April.

[27] Quah, Danny. (1993), "Galton's Fallacy and Tests of the Convergence Hypothesis", The Scandinavian Journal of Economics, 95(4):427-443, December.

[28] Quah, Danny. (1994), "Convergence across Europe", Working paper, Economics Department, LSE, London, June.

[29] Quah, Danny. (1994), "Exploiting Cross Section Variation for Unit Root Inference in Dynamic Data", Economics Letters, 44(1):9-19, January.

[30] Quah, Danny. (1994), "Ideas Determining Convergence Clubs", Working paper, Economics Department, LSE, London, September.

[31] Quah, Danny. (1994), "One Business Cycle and One Trend from (Many,) Many Disaggregates", European Economic Review, 38(3/4):605-613, April.

[32] Quah, Danny. (1995), "International Patterns of Growth: II. Persistence, Path Dependence, and Sustained Take-off in Growth Transition", Working paper, LSE, January.

[33] Quah, Danny. (1996), "Convergence Empirics Across Economies with (Some) Capital Mobility", Journal of Economic Growth. forthcoming.

[34] Romer, Paul M. (1994), "The Origins of Endogenous Growth", Journal of Economic Perspectives, 8(1):3-22, Winter. 
[35] Sala-i-Martin, Xavier. (1995), "Regional Cohesion: Evidence and Theories of Regional Growth and Convergence", European Economic Review. forthcoming. 
Table 1: Monte Carlo results $\dagger$ Convergence rate estimates

\begin{tabular}{|c|ccc|ccc|ccc|}
\hline \hline & \multicolumn{7}{|c|}{ Mean (Std. Dev.) [Frac. Rej.] } \\
\hline$N$ & \multicolumn{3}{|c|}{$T=40$} & \multicolumn{3}{|c|}{$T=50$} & \multicolumn{3}{|c|}{$T=60$} \\
\hline 10 & 0.140 & $(0.58)$ & {$[0.24]$} & 0.066 & $(0.38)$ & {$[0.27]$} & 0.027 & $(0.24)$ & {$[0.30]$} \\
20 & 0.119 & $(0.54)$ & {$[0.17]$} & 0.064 & $(0.37)$ & {$[0.20]$} & 0.023 & $(0.20)$ & {$[0.23]$} \\
30 & 0.096 & $(0.49)$ & {$[0.12]$} & 0.052 & $(0.32)$ & {$[0.16]$} & 0.024 & $(0.21)$ & {$[0.18]$} \\
40 & 0.089 & $(0.47)$ & {$[0.09]$} & 0.041 & $(0.30)$ & {$[0.12]$} & 0.016 & $(0.17)$ & {$[0.15]$} \\
50 & 0.068 & $(0.41)$ & {$[0.07]$} & 0.039 & $(0.28)$ & {$[0.11]$} & 0.014 & $(0.16)$ & {$[0.13]$} \\
100 & 0.028 & $(0.26)$ & {$[0.02]$} & 0.016 & $(0.18)$ & {$[0.04]$} & 0.007 & $(0.10)$ & {$[0.06]$} \\
200 & 0.004 & $(0.09)$ & {$[0.00]$} & 0.003 & $(0.06)$ & {$[0.01]$} & 0.002 & $(0.04)$ & {$[0.01]$} \\
\hline \hline
\end{tabular}

† These are from 10,000 independent Monte Carlo draws on cross-sectionally independent zero-drift Gaussian random walks with innovations having variance $T^{-1}$. Std. Dev. denotes the sample standard deviation and Frac. Rej. denotes the fraction rejected for machine accuracy (see text discussion). 
Table 2: Real GDP per capita (relative to world average)

First order, time-stationary, 1962 to $1984^{*}$

States: 5

\begin{tabular}{|l|ccccc|}
\hline \hline (Number) & \multicolumn{5}{|c|}{ Upper Endpoint: } \\
\hline$(456)$ & $1 / 4$ & $1 / 2$ & 1 & 2 & $\infty$ \\
$(643)$ & 0.97 & 0.03 & & & \\
$(639)$ & 0.05 & 0.92 & 0.04 & & \\
$(468)$ & & 0.04 & 0.92 & 0.04 & \\
$(508)$ & & & 0.04 & 0.94 & 0.02 \\
\hline Ergodic & 0.24 & 0.18 & 0.16 & 0.16 & 0.27 \\
\hline \hline
\end{tabular}

* The grid is empirically chosen as the indicated levels, relative to the world average. The cells are arrayed in increasing order, with the lower right-hand corner displaying transitions from rich to rich. Cell entries that are zero to two decimal places are omitted. The numbers in parentheses on the left are the number of country/year pairs beginning in a particular cell. The ergodic distribution is computed as the left eigenvector corresponding to the (isolated) unit eigenvalue. 
Table 3: (Log) personal per capita income (relative to US average) First order, time-stationary, 1948 to $1989^{*}$

Grid: Empirical uniform; States: 5

\begin{tabular}{|l|ccccc|}
\hline \hline \multirow{2}{*}{ (Number) } & \multicolumn{5}{|c|}{ Upper Endpoint: } \\
\hline$(426)$ & -0.230 & -0.110 & -0.018 & 0.080 & 0.574 \\
$(422)$ & 0.92 & 0.08 & & & \\
$(421)$ & 0.06 & 0.84 & 0.10 & & \\
$(422)$ & & 0.09 & 0.81 & 0.09 & \\
$(421)$ & & & 0.10 & 0.85 & 0.04 \\
\hline Ergodic & 0.19 & 0.22 & 0.23 & 0.20 & 0.16 \\
\hline \hline
\end{tabular}

* The grid is, as indicated, empirically chosen to give a uniform distribution over the observed sample. See other notes to table 2. 

Figure 3.1: $\sigma$-divergence towards $\sigma$-convergent steady state

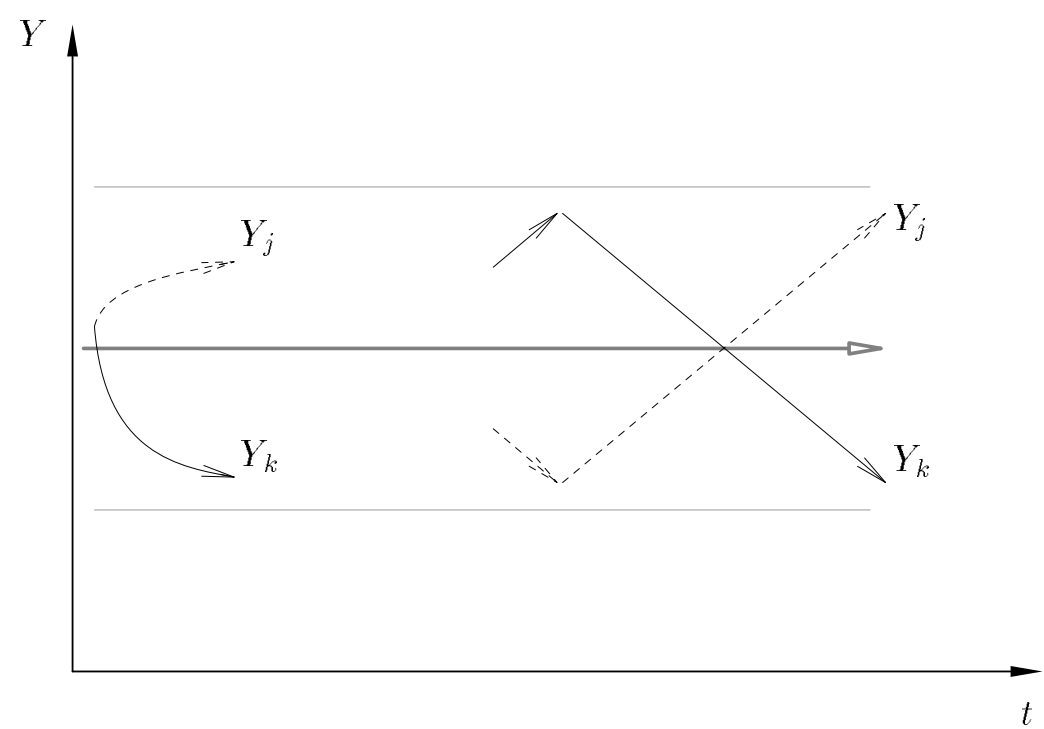


Figure 3.2: Coincident $\beta$ and $\sigma$ convergence

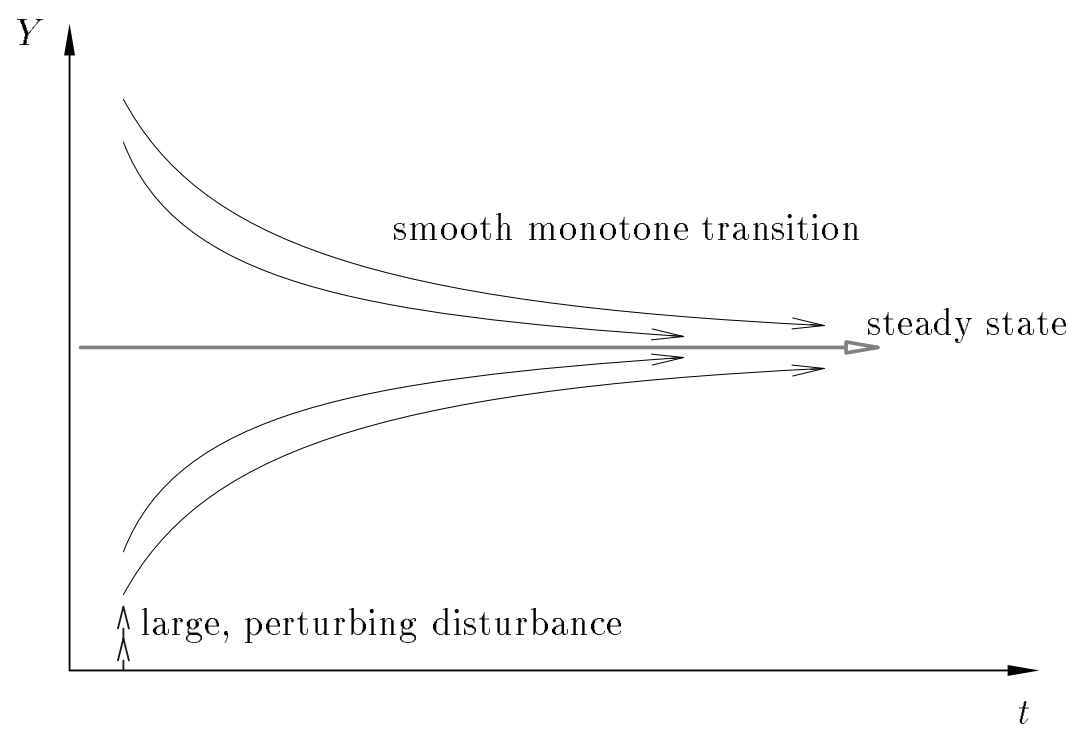


Figure 3.3: $\sigma$-convergent limit with criss-crossing

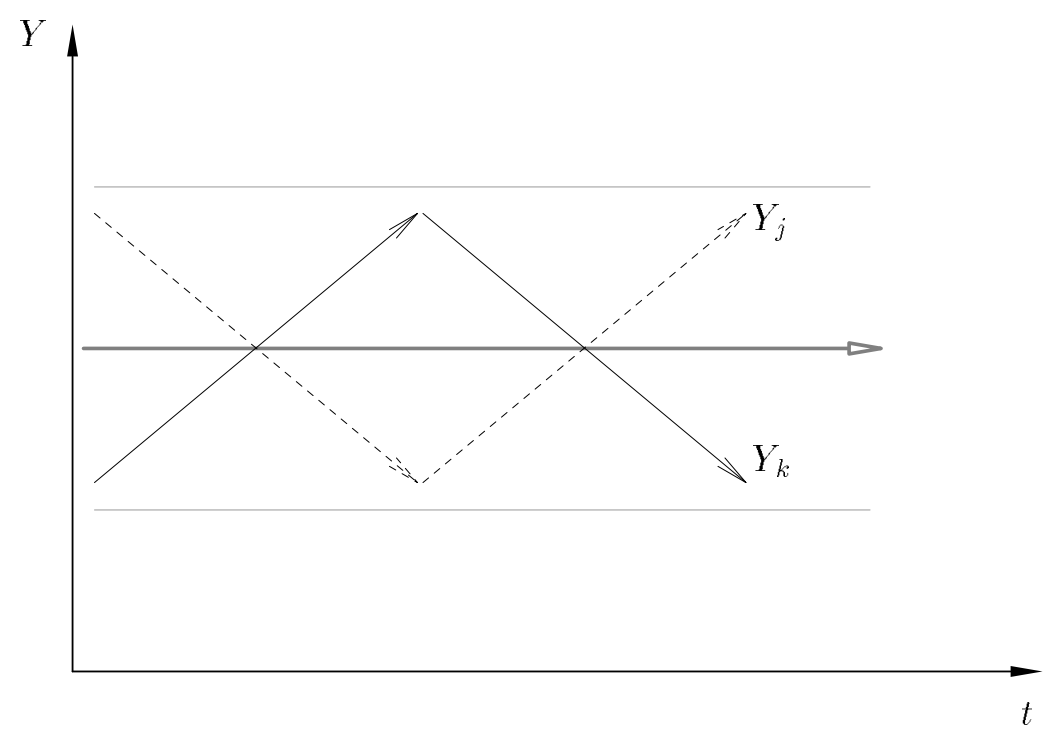


Figure 3.4: $\sigma$-convergent limit with persistent inequality

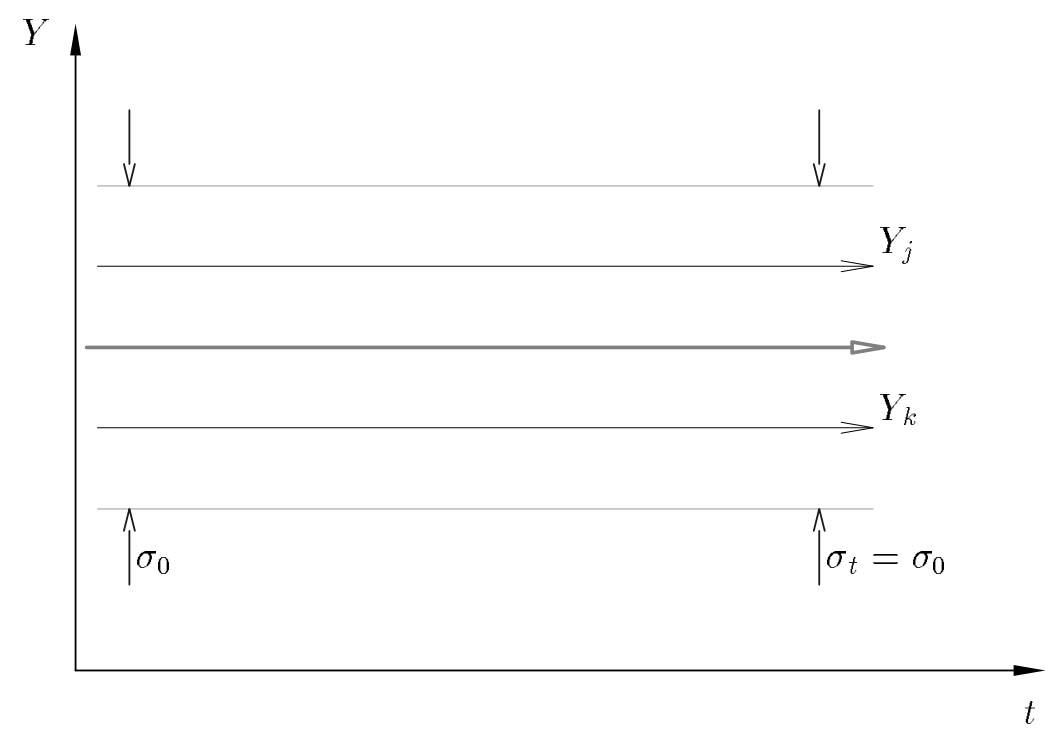


Figure 4.1: Evolving distributions, tending towards bimodal increasing income values

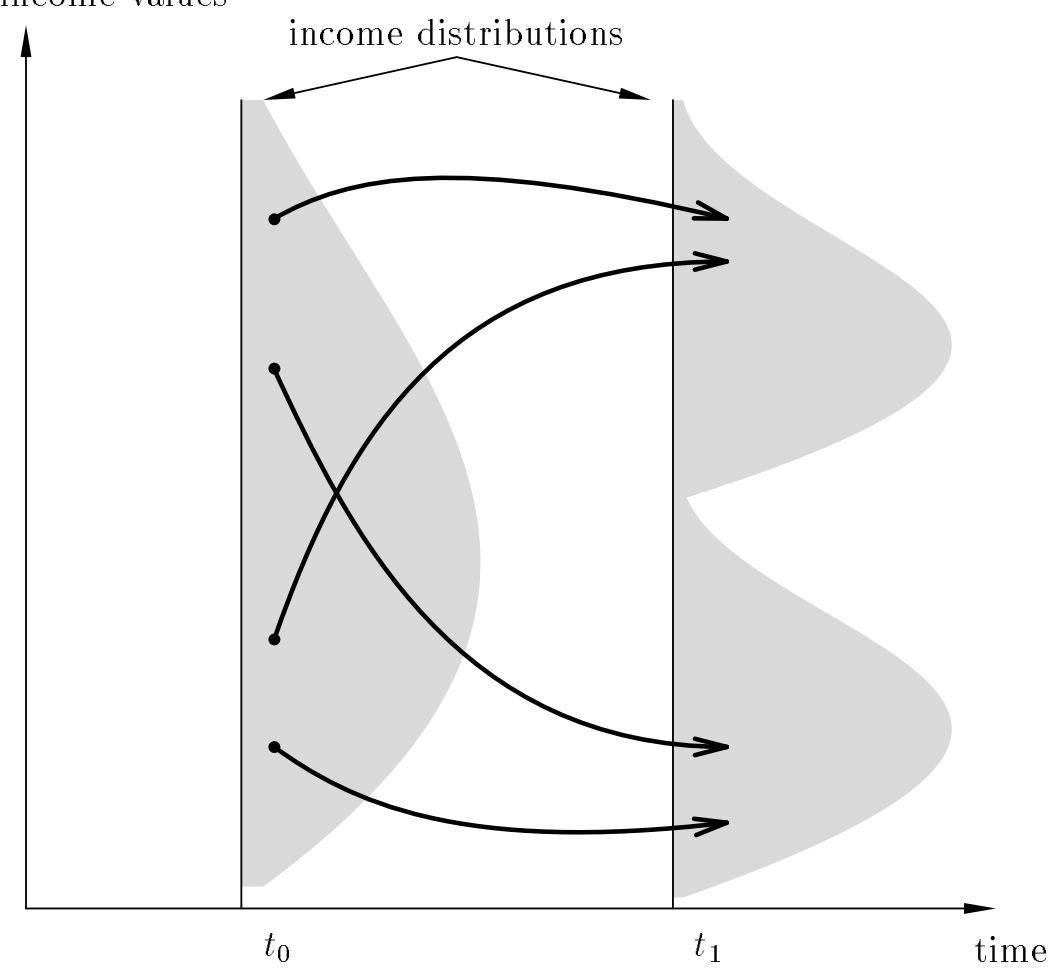

